Physical Sciences | Cher Ming Tan

\section{Physics of degradation}

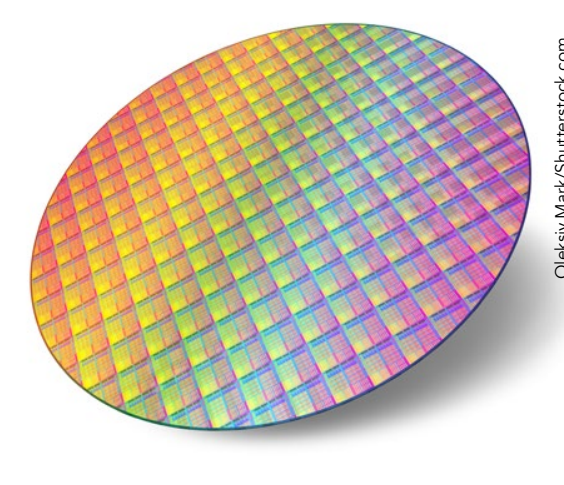

The difference between reliability engineering and reliability science

\section{$\bigvee \begin{aligned} & \text { e have probably all bought } \\ & \text { a piece of equipment based } \\ & \text { on certain expectations }\end{aligned}$ nere quickly disappointed by the quality of the product received or the reliability of its performance. The majority of consumers nowadays check for reviews before purchasing a product rem a serice, which makes reviews the ellers. Negative reviews can affect the sales of a product significantly especilly those focusing on poor quality or unreliable performance. While quality can be controlled in a \\ ALTERNATIVE WAYS TO ENSURE RELIABILITY Reliability can be described as quality over time, or the probability of a product to continue performing as advertised after an extended time or use. Especially for products that are expected to maintain their performance over a conditions, reliability is key. An import category of such products is electronics, lamps, which find wide application in many products of our everyday life. Since it is impractical to check every}

Quality and reliability are key factors for a product's commercial success. Reliability testing usually occurs at the final product, using destructive methods. This implies that there might be added costs and delays if reliability tests fall. Looking at reliability sciences from the physics of Ming Tan from the Research Center on Reliability Sciences and Trof Cher in Taiwan is proposing an innovative approach. He studies the reliability of products based on a physics, chemistry and materials sciences level, before the assembly of the final product. Combining experimental data and simulations, he can predict the reliability of the final product under various operational conditions, saving precious time and money.

Towards this direction, a niche research rea has recently emerged, studying the physics of fallures and material sciences and determine reliability statistics. This is the area of reliability sciences, where experimentation, multi-scale simulation, and atomic finite element modelling are used in parallel, in an effort to develop methods and predicting reliability of components before the assembly of the final product. In this way, the reliability of a product will be determined beforehand, allowing for faster and cheaper

modifications, before 'final' products are developed. This would mean that current reliability measurements, when performed on the final product, would act as secondary measurements, confming the initially predicted reliability.

EXPLORING DEGRADATION

\section{MECHANISMS}

In an effort to find the balance between of products, their cost, and their reliability, Prof Cher Ming Tan, a pioneer in the manufacturing processes, improved design), reliability is not so easy to be controlled. The reason for that is the inability to check every single product for Us performance, as reliability tests surve paradigm from testing on final products to reliability predictions on individual products based on the exploration of the underlying physics and chemistry of material degradation. single semiconductor and LED lamp for their reliability, a new way of ensuring on the focusing on reliability studies order to reduce costs ry of a product, in

Stress-Induced Voiding
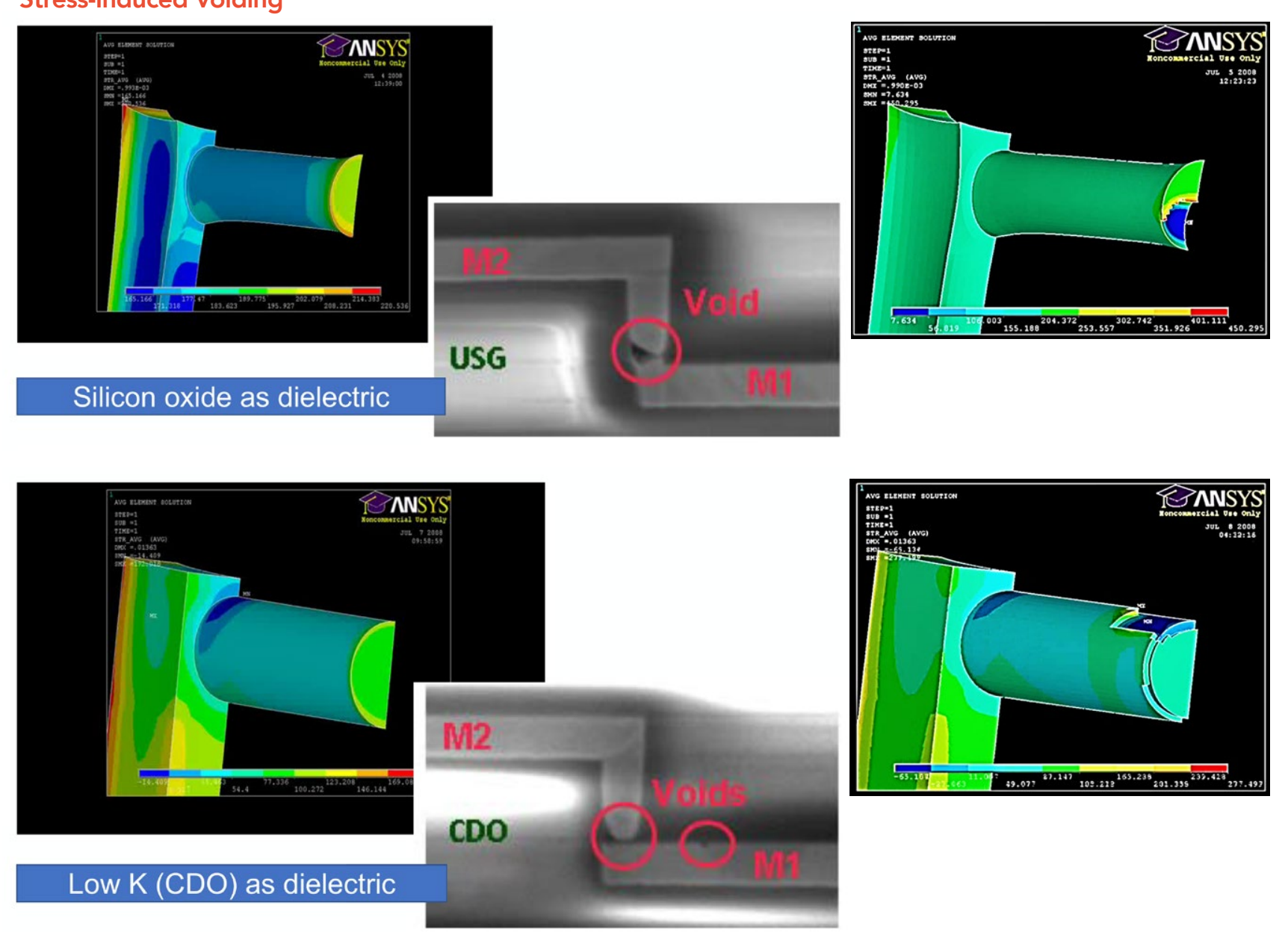

Atomic simulation of stress-induced voiding using finite element metho
images) and low $\mathrm{k}$ (bottom images). Left: initial; right: after some time.

of products or their parts, launch delays, availability, or potential need to recall faulty items.

According to Prof Tan, the critica challenges of ensuring product reliability fast results, and highly reliable products. These goals are contradictory to one another, since fast results usually imply high costs, high reliability also means increased product cost, and when companies try to reduce product costs, this usually results in lower quality and reliability. Prof Tan's vision has been to shift the paradigm of reliability testing from testing on final products to reliability predictions/computation on individual products based on the exploration of the underlying physics and chemistry of material degradation. He beleves that identifying the degradation mechanisms and the physics of degradation on the material at atomic level, and scaling up finite element modelling, atomic-

based material simulation, and density function theory formulation, can lead to prediction, or rather computation of the time and money in the long run.

TESTING THE BRIGHTNESS OF LEDS Prof Tan conducted research using products in an effort to whidely applied innovative approach to reliability and establish reliability sciences. Using LEDs and semiconductors as examples, he and his team explored the reasons behind common degradation mechanisms of such devices, through experimen combined with simulations that confirmed the suggested mechanisms of Prof Pef explaining about the need for increased lumen flux of white LEDs (basically how bright and white their light shines), and and change of the light from white to a warmer hue This chan wh whe to a to the thickness and concentration of the phosphor layer incorporated into the LED design. It was shown that with the brightness of the LED will incion up to a point where temperature rise and scattering effects will overpower the brightness increase and compromise investigation on the reasons behind such behaviour, it was indicated experimentally and further supported through computational work that backscattering of light in higher presence of phosphor particles led to reduced performance. Results from this work could also be used te explan and predict the performance of teDs enclosed in a casing, as opposed to (a)

Another article looking into LED's performance focused on the degradation to the effects of moisturs application, due on the silis of casing Results showed 

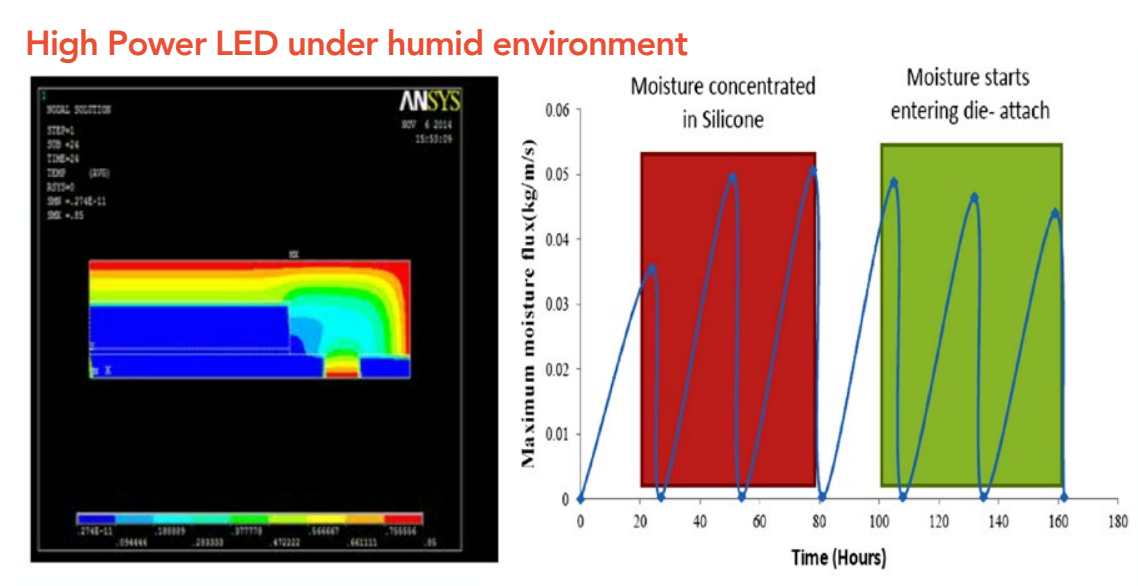

Prof Tan studied the degradation of outdoor LEDs by examining moisture and ten
site or at the die attach (the process of attaching the semiconductortor to a packagel

The critical challenges of ensuring product reliability are the simultaneous needs for low cost, fast results, and highly reliable products.

that the casing was getting discoloured over time, a fact that led to changes in the
brightness of the LED. Discolouration was attributed to four different mechanisms of silicone ageing, which due to the presence of moisture and increased temperature were accelerated. Prof Tan's work shed light (pun intended) into the way slicone degradation discolouration occurred (hydrolysis was the main cause of ageing, and thermal oxidation was the main cause of discolouration) and how material degradation affected the LED performance, hence its reliability

\section{THE CHEMISTRY AND PHYSICS} OF SEMICONDUCTOR FALURE Besides LEDs, Prof Tan also

examined the physics of degradation and degradation mechanisms semiconductor reliability from severa different angles. He examined the resistance of semiconductor parts to moisture on a chemistry and materials level, developing an understanding that could lead to the prediction or the reliability of such products without the need to conduct a humidity test on the final product. By identifying functiona groups (a chemical term for groups that are prone to react with other groups, such as those of water, and change the substance of the material) and exploring testing approach was destoped, in effort to avoid destructive assessment. In this case, knowing the performance of the materials used to build the semiconductor and its package, its reliability could be predicted and/or final product, avoiding the need for further testing.

Past moisture resistance of semiconductor casings, Prof Tan also chips, and how it could compromise the reliability. Using advanced microscopy techniques, it was revealed that the circuit was melting upon extended use of and more in-depth analysis led to indications on the explanation of the failure mechanisms. Using simulations to complement experimental data. Prof Tan and his team were able to create $3 \mathrm{D}$ models of the chips and examine the magnetic field distribution on the chip. They revealed that additional electrical current was produced due to electromagnetic effects, leading to increased temperature and the subsequent melting of the circuit materials, which caused failure of the chip, due to a mechanism different to the already identified ones. This new the material science perspective could be used to improve the design of chips looked into the design circuit of $5 \mathrm{G}$ information on failure mechanisms from
FROM ELECTROMIGRATION TO MATERIAL DAMAGE Prof Tan's work in reliability sciences ectromigration as a reason foilure of integrated interconnections (the electrical connections between part of a circuit). Electromigration can be described as the movement of atoms in a material structure when material is put under some form of stress, for example when electricity is passing through it. This movement can lead to tiny, naturally existing voids within the material structure eventually getting connected due to atom movement, forming bigge voids that can compromise the stability of damage of the circut.

Studying the time to failure of integrated interconnections due to electromigration Prof Tan combined experimental

analysis and a novel simulation approach and proposed three stages during electromigration that lead to failure. These were the incubation of tiny voids, their nucleation (build-up), and finally, their growth. An interesting follow-up to this work was an investigation of failure cause by stress migration, where Prof Tan examined through simulations the factors affecting void nucleation and growth in different materials under stress. He concluded that stress gradient and effective bulk modulus (an expression of the extent of how the volume of a materi changes under pressure) were the two affected stress-induced voiding

\section{THE FUTURE OF}

In a nutshell, Prof Tan's contribution

in reliability sciences towards the

explanation of failure and degradation

mechanisms using a combination of

experiments and multi-scale simulation

has been pivotal for this emerging

discipline. With the increase of product design complexity, product cost, and need for improved quality and reliability, assessment of reliability is absolutely necessary and should not be discounted. Introducing ways of assessing reliability a an earlier level, implementing assessment on the level of physical, chemical, and material sciences, before the final product assembly, can improve cost and
time efficiency.

\section{Behind the Research}

\section{Professor Cher Ming Tan}

E: cherming@ieee.org E: cmtan@cgu.edu.tw T: +88632118800 ext. 5952
W: http://www.crest.cqu.edu.tw W: Wttp://www.chermingtan.com

Research Objectives

Prof Tan explores the underlying physics and chemistry of material degradation to evaluate products for their reliability.

\section{Detail}

Chang Gung University

259 Wen Hua 1 st Road

Kwei-Shan, Taoyuan

Taiwan

Bio

Professor Cher Ming Tan received his PhD in Electrica Engineering from the University of Toronto in 1992. He from 1996 until 2014 . Tn then joined Chang Gupg University in Taiwan and set up the Research Center Reliability Sciences and Technologies where he currently acts as Director.

\section{Funding}

Chang Gung University
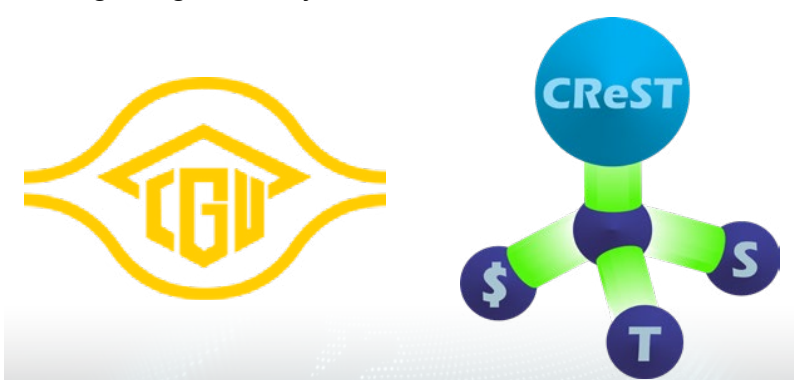

\section{References}

Tan, C.M., ..., Kuo, H.C. (2018). Physical Limitations of Phosphor layer thickness and concentration for White LEDs. SCIENTIFIC REPORTS, 8, 2452

Singh P. and Tan C.M. (2018). Time evolution of packaged LED lamp degradation in outdoor applications. Optical Materials, 86, 148-154.

Tan C.M., ..., Chen Y.J. (2020). Moisture resistance evaluation on single electronic package moulding C, 8, 1943-

Sangwan V., ..., Chiu H.C. (2020). Electromagnetic Induced Failure in GaN-HEMT High-Frequency Power Amplifier. IEEE TRANSACTIONS ON INDUSTRIAL ELECTRONICS, 67(7), 5708-5716.

Li, W.,..., Raghavan, N. (2009). Dynamic simulation of void nucleation during electromigration in narrow integrated circuit interconnects. J. Appl. Phys., 105, 014305.

Hou, Y. and Tan, C.M. (2009). Comparison of stressinduced voiding phenomena in copper line-via structures with different dielectric materials. Semicond. Sci. Technol, 24,085014

Personal Response
How could reliability sciences find application in
industry?
II Reliability is important to industry, and reliability
science can help companies to design the products for a
specific reliability by choosing appropriate materials and
structures. Reliability science can also help companies
to ensure their individual product reliability without
destructive testing and long test time.
How much time would the creation of a material
database take, and could it be used as a simulation
matrix to predict product reliability?
IU The initial creation of material database can take 1-2
months' time, and once it is set up, it can be used for
future reliability computation and prediction until where
new materials are employed.

